\title{
Teste de condutividade elétrica para avaliação do potencial fisiológico de sementes de canola
}

\author{
Marília Milani, ${ }^{1, *}$, Nilson de Lemos Menezes², Sidinei José Lopes ${ }^{2}$
}

\begin{abstract}
RESUMO
O objetivo deste trabalho foi determinar o volume de água e o período de imersão a serem utilizados no teste de condutividade elétrica, para avaliar o potencial fisiológico de sementes de canola. Foram utilizados quatro lotes de sementes, os quais foram inicialmente caracterizados, quanto à qualidade, por meio dos testes de germinação, primeira contagem, comprimento de plântulas e emergência em areia. O teste de condutividade elétrica foi realizado a $25^{\circ} \mathrm{C}$, por períodos de: 2, 4, 8, 16 e 24 horas de imersão, em 25 e $50 \mathrm{~mL}$ de água deionizada. O delineamento experimental utilizado foi inteiramente casualizado, com os tratamentos em esquema fatorial 4 × 2 x 5 (quatro lotes, dois volumes de água e cinco períodos de imersão em água), com quatro repetições de 50 sementes. O teste de condutividade elétrica é eficiente para a avaliação do potencial fisiológico de sementes de canola, sob a condição de 50 sementes imersas em 25 $\mathrm{mL}$ de água deionizada por 8 horas.
\end{abstract}

Palavras-chave: Brassica napus L. var. oleifera, teste de vigor, qualidade de sementes.

\section{ABSTRACT}

\section{Electrical conductivity test for the evaluation of physiological potential of seed lots of canola}

This work aimed to determinate the best water volume and immersion period used on electrical conductivity test for the evaluation of physiological potential of seed lots of canola. Four seed lots of canola were evaluated, which were firstly characterized with respect to quality through tests of germination, first germination count, seedling length and emergence on sand. The electrical conductivity test was carried out at $25^{\circ} \mathrm{C}$. Seeds were imbibed in 25 and $50 \mathrm{~mL}$ of distilled water during 4, 8, 16 and 24 hours. The experimental design used was completely randomized, with four replicates of 50 seeds. Data was analyzed in a factorial scheme with four seed lots, two water volumes and five immersion periods. The electrical conductivity test carried out under immersion of 50 seeds during 8 hours in $25 \mathrm{~mL}$ at $25^{\circ} \mathrm{C}$ of deionized water is efficient to evaluate the physiological potential of seed lots of canola.

Key words: Brassica napus L. var. oleifera, vigor test, seed quality.

\footnotetext{
Recebido para publicação em 06/01/2011 e aprovado em 09/04/2012

${ }^{1}$ Engenheira-Agrônoma, Mestre. Departamento de Biologia, Centro de Ciências Naturais e Exatas, Universidade Federal de Santa Maria, Av. Roraima, 1000, Cidade Universitária, Bairro Camobi, 97105-900, Santa Maria, Rio Grande do Sul, Brasil. mariliakmilani@gmail.com (*autora para correspondência).

${ }^{2}$ Engenheiros-Agrônomos, Doutores. Departamento de Fitotecnia, Centro de Ciências Rurais, Universidade Federal de Santa Maria, Av. Roraima, 1000, Cidade Universitária, Bairro Camobi, 97105-900, Santa Maria, Rio Grande do Sul, Brasil.nlmenezes@smail.com.br, sjlopes@pq.cnpq.br
} 


\section{INTRODUÇÃO}

A canola (Brassica napus L. var. oleifera) pertence à família Brassicaceae e possui entre 45 e 50\% de óleo, no grão, e de 34 a 38\% de proteína, no farelo (Tomm, 2007). É uma boa opção para compor sistemas de rotação de culturas ou para ser utilizada como cobertura vegetal do solo no período de inverno, no sul do Brasil (Baier \& Roman, 1992).

O óleo pode ser utilizado como biodiesel, uma alternativa ambientalmente sustentável, que reduz a emissão de $\mathrm{CO}_{2}$, materiais particulados e hidrocarbonetos não queimados, minimizando, com isso, o impacto do efeito estufa (Lima Neto et al., 2006). Para isso, tem-se estimulado a utilização de biocombustíveis renováveis, provenientes de espécies agrícolas oleaginosas, como, por exemplo, a canola.

Para implementar a canola no sistema de produção agrícola nacional, é fundamental que sejam produzidas sementes de elevada qualidade e que sejam estabelecidos métodos padronizados na avaliação da qualidade fisiológica das sementes (Ávila et al., 2005). Essa qualidade é determinada por sua capacidade em desempenhar funções vitais, as quais são caracterizadas pela germinação, vigor e longevidade (Bewley \& Black, 1994).

O teste de germinação é o procedimento oficial para avaliar a capacidade das sementes de produzirem plântulas normais em condições ideais, mas nem sempre revela diferenças de desempenho entre lotes de sementes durante o armazenamento ou, em campo (Carvalho \& Nakagawa, 2000).

Dessa maneira, é importante avaliar as sementes por meio dos testes de vigor, como complemento às informações fornecidas pelo teste de germinação, pois esses são mais sensíveis à avaliação da qualidade fisiológica, indicando eventos metabólicos que antecedem a perda da germinação, os quais servem como base para avaliar o vigor. Porém, quanto mais distante da perda do poder germinativo estiver este evento, mais sensível será o teste (Marcos Filho, 1999).

O teste de condutividade elétrica baseia-se no fato de que o vigor está diretamente relacionado com a integridade do sistema de membranas celulares. Sendo assim, sementes menos vigorosas (ou mais deterioradas) apresentam menor velocidade de restabelecimento da integridade das membranas celulares durante a imersão, e, em consequência, liberam maiores quantidades de solutos para o meio exterior, tais como: açúcares, aminoácidos, ácidos orgânicos, proteínas e substâncias fenólicas, e de íons inorgânicos: $\mathrm{K}^{+}, \mathrm{Ca}^{++}, \mathrm{Mg}^{++}, \mathrm{Na}^{++}$(Marcos Filho, 2005).

Além disso, o teste de condutividade elétrica apresenta as principais características que um teste de vigor deve ter, entre as quais podem-se destacar simplicidade, baixo custo, objetividade e rapidez, fornecendo informações em até 24 horas (Marcos Filho, 1999).

No entanto, o teste de condutividade elétrica tem sua padronização apenas para sementes de ervilha, nas Regras Internacionais para Análise de Sementes (Ista, 2006). Existe, portanto, a necessidade de estudar e analisar a aplicação e padronização deste teste em outras culturas, para avaliar o potencial fisiológico das sementes.

O objetivo do trabalho foi determinar as condições adequadas, quanto ao volume de água e ao período de imersão, para o teste de condutividade elétrica, na avaliação do potencial fisiológico de sementes de canola.

\section{MATERIAL E MÉTODOS}

O experimento foi realizado no Laboratório Didático e de Pesquisa em Sementes, do Departamento de Fitotecnia, na Universidade Federal de Santa Maria. Utilizaram-se quatro lotes de sementes de canola, não tratados, do cultivar PFB-2, os quais foram inicialmente caracterizados quanto à qualidade, por meio dos testes de germinação, primeira contagem, comprimento de plântulas e emergência em leito de areia.

O teste de germinação foi realizado com quatro subamostras de 50 sementes para cada lote, semeadas em papel toalha, umedecido com água destilada, na proporção de 2,5 vezes a massa do papel seco. O teste foi conduzido à temperatura de $20^{\circ} \mathrm{C}$, sendo as contagens realizadas no quarto e sétimo dia, após a semeadura, e os resultados expressos em percentagem de plântulas normais. $\mathrm{O}$ critério utilizado para avaliação das estruturas essenciais das plântulas, que determinaram serem consideradas normais, foi segundo Brasil (2009).

A primeira contagem foi realizada juntamente com o teste de germinação, determinando-se a percentagem de plântulas normais, no quarto dia após a instalação do teste, e os resultados foram expressos em percentagem de plântulas normais.

Para comprimento de plântulas, avaliou-se o comprimento médio de 10 plântulas normais de cada lote, escolhidas aleatoriamente, obtidas a partir da semeadura de quatro subamostras de 15 sementes por lote, no terço superior do papel toalha. Os rolos de papel, contendo as sementes, permaneceram por cinco dias em germinador à temperatura de $20^{\circ} \mathrm{C}$, quando se avaliou o comprimento total das plântulas, com o auxílio de uma régua graduada em milímetros. O resultado utilizado foi a soma das médias dos comprimentos da parte aérea e da raiz, conforme descrito por Nakagawa (1999), expressos em centímetros.

A emergência em leito de areia foi realizada com adaptação da metodologia descrita por Nakagawa (1994), com quatro subamostras de 50 sementes para cada lote. A areia 
média utilizada foi previamente peneirada, lavada e colocada em bandejas de plástico. Para semeadura, foram abertos sulcos longitudinais em cada bandeja, com $2 \mathrm{~cm}$ de profundidade e espaçados de $3 \mathrm{~cm}$ entre si, utilizando-se 50 sementes por sulco.

O teste foi realizado em ambiente de laboratório, sendo a umidade mantida por meio de irrigações frequentes. No décimo quarto dia após a semeadura, quando não foi mais observada emergência de novas plântulas, avaliouse a percentagem de plântulas normais, conforme as recomendações contidas nas Regras para Análises de Sementes (Brasil, 2009).

Os tratamentos utilizados para padronizar o teste de condutividade elétrica foram organizados em esquema trifatorial: 4 × 2 × 5 (quatro lotes, dois volumes de água e cinco períodos de imersão em água). Foram estudadas variações na quantidade de água deionizada, 25 e $50 \mathrm{~mL}$ e, no período de imersão: 2, 4, 8, 16 e 24 horas. Avaliaram-se quatro subamostras de 50 sementes, fisicamente puras, pesadas com precisão de duas casas decimais $(0,01 \mathrm{~g})$. Em seguida, as sementes foram imersas em $25 \mathrm{~mL}$ e $50 \mathrm{~mL}$ de água deionizada e mantidas em câmara tipo BOD à $25^{\circ} \mathrm{C}$, durante os cinco períodos de imersão. Após cada período de imersão, a condutividade elétrica da solução foi determinada por meio de leitura em condutivímetro DIGIMED, CD-21, com os resultados expressos em $\mu \mathrm{S} . \mathrm{cm}^{-1}$. $\mathrm{g}^{-1}$ de semente. À exceção dos fatores estudados, o teste foi conduzido conforme descrito por Vieira \& Krzyzanowski (1999).

Para as variáveis de caracterização inicial dos lotes e da condutividade elétrica, procedeu-se a análise de variância e teste de Tukey para comparação das médias, a $5 \%$ de probabilidade. Para o estudo da condutividade elétrica, no esquema trifatorial, considerou-se o efeito das interações, com o uso da regressão polinomial para o fator tempo de imersão em água. Os dados em percentagem (x) foram transformados em arco seno da raiz quadrada de $\mathrm{x} / 100$.

Foi realizada a análise de correlação simples entre os valores do teste de condutividade elétrica, nos volumes de água de 25 e $50 \mathrm{~mL}$, e os testes de germinação, da primeira contagem da germinação, do comprimento de plântula e da emergência em areia. O nível de significância adotado foi de $5 \%(\mathrm{p}<0,05)$. As informações foram processadas, utilizando-se o programa estatístico Soc (NTIA/ EMBRAPA, 1989).

\section{RESULTADOS E DISCUSSÃO}

Pela caracterização da qualidade inicial dos lotes (Tabela 1), observa-se que o teste de germinação não estratificou os lotes 1,2 e 3 em diferentes níveis; apenas o lote 4 apresentou diferença significativa dos demais, com a menor percentagem de germinação.
Os testes de vigor, como primeira contagem da germinação, comprimento de plântula e emergência em areia, foram mais sensíveis ao avaliar a qualidade das sementes dos diferentes lotes, identificando diferenças de qualidade entre lotes, que o teste de germinação não havia identificado. Além de aqueles testes apresentarem maior discriminação dos lotes do que o teste de germinação, também há que se considerar que a diferenciação pelo teste de médias depende da precisão com que foi conduzido cada teste, ou seja, do erro experimental e do erro de medição de cada variável. O único resultado comum a todos os testes foi o do lote 4 , que sempre foi classificado como de qualidade inferior.

Diferenças no comportamento de lotes com germinação semelhante estão associadas ao fato de que os primeiros sinais de deterioração ocorrem antes da perda da capacidade germinativa (Delouche \& Baskin, 1973), que, mesmo sendo um importante indicador de perda da viabilidade, é, porém, o último parâmetro observado antes da morte da semente. $\mathrm{O}$ teste de germinação é eficiente pelo menos por dois aspectos: fornece informações sobre o potencial de uma amostra para germinar, sob condições ótimas de ambiente, e é padronizado. Mesmo assim, é considerado incompleto, pela possível superestimativa do potencial fisiológico das sementes (Marcos Filho, 1999).

O teste de emergência em areia, realizado em condições não muito distantes ou diferentes das condições de campo, foi o único teste que estratificou os quatro lotes em quatro níveis de potencial fisiológico, ou seja, maior vigor para o lote 1 e, na sequência, até o pior lote, o lote 4 , que não teve emergência de plântulas até o momento da avaliação.

Caracterizados os lotes, a condutividade elétrica, avaliada sob os três fatores (volume de água, período de imersão e lotes), apresentou interação tripla significativa ( $p<0,05)$, ou seja, os lotes de sementes têm um desempenho diferente em cada combinação de volume de água e período de imersão.

Observa-se que a menor diluição dos lixiviados (50 sementes em $25 \mathrm{~mL}$ ) diferencia a qualidade dos lotes, em

Tabela 1. Caracterização da qualidade inicial (valores médios) de quatro lotes de sementes de canola quanto à germinação $(\mathrm{G})$, primeira contagem (PC), comprimento de plântula (CP) e emergência em areia (EA)

\begin{tabular}{ccccc}
\hline Lotes & G (\%) & PC (\%) & CP (cm) & EA (\%) \\
\hline 1 & $94 \mathrm{a}^{*}$ & $70 \mathrm{~b}$ & $8,15 \mathrm{a}$ & $81 \mathrm{a}$ \\
2 & $95 \mathrm{a}$ & $87 \mathrm{a}$ & $4,48 \mathrm{~b}$ & $62 \mathrm{~b}$ \\
3 & $96 \mathrm{a}$ & $91 \mathrm{a}$ & - & $9 \mathrm{c}$ \\
4 & $69 \mathrm{~b}$ & $28 \mathrm{c}$ & - & $0 \mathrm{~d}$ \\
\hline CV & $11,30 \%$ & $11,68 \%$ & $24,64 \%$ & $11,68 \%$ \\
\hline
\end{tabular}

* Médias seguidas pela mesma letra na coluna não diferem entre si, pelo teste de Tukey, a 5\% de probabilidade. - = ausência de plântulas emersas. 
tempo menor de imersão do que a maior diluição (50 sementes em $50 \mathrm{~mL}$ ), e que, em 2 horas, já caracteriza de forma idêntica ao teste de germinação aplicado aos lotes (Tabela 2), ou seja, o lote 4 como o menos vigoroso.

Na figura 1 , observa-se que a concentração de lixiviados é maior em $25 \mathrm{~mL}$ do que em $50 \mathrm{~mL}$, o que corrobora os resultados de Dutra \& Vieira (2006) e de Vidigal et al. (2008). Também se observa que a concentração de lixiviados aumenta durante o tempo de imersão, conforme relatado por Mello et al. (1999), em sementes de brócolos; Vieira et al. (1996), em sementes de feijão e soja; e Vanzolini \& Nakagawa (1998), em sementes de amendoim.

O efeito da diluição parece ser diferente, entre os lotes de qualidades diferentes, e a própria tendência da condutividade elétrica com o tempo de imersão em água propicia o ajuste de modelos polinomiais lineares, para os lotes de maior vigor, e, quadráticos, para os lotes de menor vigor (Figura 1). Isso se explica por ser intensa a liberação inicial dos lixiviados, tanto pelas sementes intactas e vigorosas, como pelas danificadas, tornando-se difícil a identificação de possíveis diferenças de qualidade entre os lotes, logo no início da imersão. No entanto, com o decorrer deste processo, a quantidade de lixiviados liberados pelas sementes vigorosas vai-se estabilizando, em razão, principalmente, da reorganização das membranas, favorecendo a ordenação dos lotes em níveis de qualidade (Menezes et al., 2007).

As membranas celulares, quando se apresentam mais íntegras ou com maior capacidade de restabelecer sua integridade, durante a imersão, liberam menor quantidade de lixiviados para o meio externo (Bewley \& Black, 1994; Vieira \& Krzyzanowski, 1999).

O aumento de $100 \%$ no volume de água (25 mL para $50 \mathrm{~mL}$ ) para o teste de condutividade elétrica da canola, no lote 3 , proporcionou uma redução de $40 \%$ no valor da condutividade elétrica, estimada no ponto de máxima das funções quadráticas, para $25 \mathrm{~mL}(21,71$ horas, 280,15 $\left.\mu \mathrm{S} . \mathrm{cm}^{-1} \cdot \mathrm{g}^{-1}\right)$ e $50 \mathrm{~mL}\left(23,20\right.$ horas, $\left.168,09 \mu \mathrm{S} . \mathrm{cm}^{-1} \cdot \mathrm{g}^{-1}\right)$.

Tabela 2. Dados médios do teste de condutividade elétrica $\left(\mu \mathrm{S} \mathrm{cm}^{-1} \mathrm{~g}^{-1}\right)$ de quatro lotes de sementes de canola, em função dos volumes de água e dos períodos de imersão

\begin{tabular}{lcccccc}
\hline Volume de & Lotes & \multicolumn{5}{c}{ Períodos de imersão (horas) } \\
\cline { 3 - 7 } água $(\mathbf{m L})$ & 1 & $\mathbf{2}$ & $\mathbf{4}$ & $\mathbf{8}$ & $\mathbf{1 6}$ & $\mathbf{2 4}$ \\
\hline & $29,68 \mathrm{a}^{*}$ & $85,56 \mathrm{a}$ & $107,92 \mathrm{a}$ & $134,25 \mathrm{a}$ & $154,81 \mathrm{a}$ \\
25 & $26,87 \mathrm{a}$ & $90,19 \mathrm{a}$ & $118,55 \mathrm{a}$ & $149,69 \mathrm{a}$ & $165,89 \mathrm{a}$ \\
& 3 & $97,09 \mathrm{a}$ & $131,78 \mathrm{ab}$ & $207,29 \mathrm{~b}$ & $254,15 \mathrm{~b}$ & $281,11 \mathrm{~b}$ \\
& 4 & $158,97 \mathrm{~b}$ & $177,85 \mathrm{~b}$ & $297,36 \mathrm{c}$ & $407,24 \mathrm{c}$ & $435,03 \mathrm{c}$ \\
\hline & 1 & $38,03 \mathrm{a}$ & $55,96 \mathrm{a}$ & $73,30 \mathrm{a}$ & $92,69 \mathrm{a}$ & $95,60 \mathrm{a}$ \\
50 & 2 & $48,41 \mathrm{a}$ & $54,05 \mathrm{a}$ & $76,95 \mathrm{a}$ & $98,08 \mathrm{ab}$ & $93,20 \mathrm{a}$ \\
& 3 & $55,84 \mathrm{a}$ & $74,31 \mathrm{a}$ & $114,16 \mathrm{ab}$ & $152,63 \mathrm{bc}$ & $168,66 \mathrm{~b}$ \\
& 4 & $63,62 \mathrm{a}$ & $90,53 \mathrm{a}$ & $164,02 \mathrm{~b}$ & $209,48 \mathrm{c}$ & $270,19 \mathrm{c}$ \\
\hline
\end{tabular}

$\mathrm{CV}=14,85 \%$

* Médias seguidas pela mesma letra na coluna não diferem entre si, pelo teste de Tukey, a 5\% de probabilidade.

$25 \mathrm{~mL} \quad$ olote $1 \mathrm{CE}=3,2104 \mathrm{x}+79,77 \mathrm{R} 2=0,9767$

$\times$ lote $2 C E=-0,2037 \times 2+9,5802 x+52,084 R 2=0,9934$

$\Delta$ lote $3 C E=-0,4628 \times 2+20,092 x+62,077 R 2=0,9858$

Dlote 4 CE $=-0,6773 \times 2+30,804 x+86,689$ R2 $=0,9893$

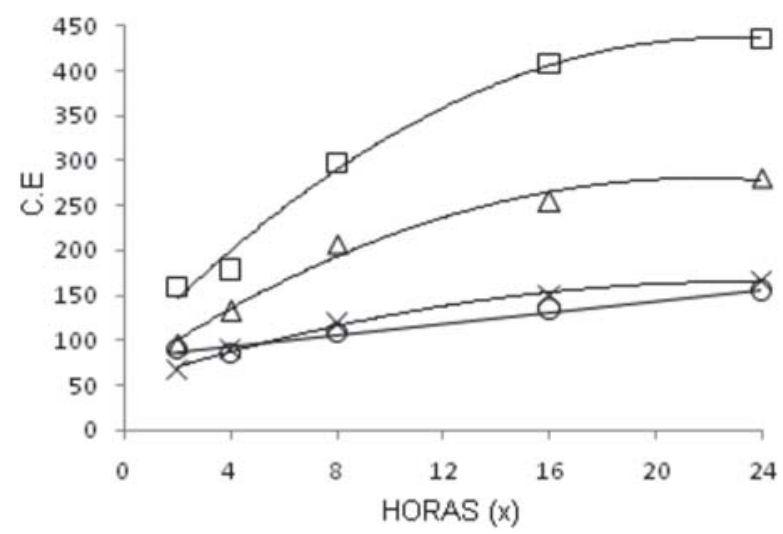

$50 \mathrm{~mL}$ Olote $1 \mathrm{CE}=2,4744 \mathrm{x}+44,393 \mathrm{R} 2=0,981$

$\times$ lote $2 C E=2,1973 x+50,408 R 2=0,9975$

$\Delta$ lote $3 C E=-0,2496 \times 2+11,579 x+33,805 R 2=0,7994$

미 te $4 C E=-0,2604 \times 2+15,815 x+36,465 R 2=0,8511$

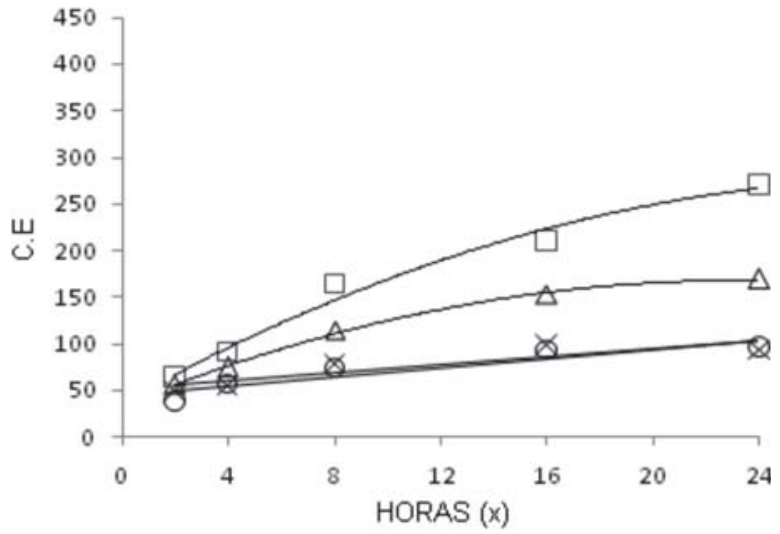

Figura 1. Condutividade elétrica (C.E) $\left(\mu \mathrm{S} . \mathrm{cm}^{-1} \cdot \mathrm{g}^{-1}\right)$ de quatro lotes de sementes de canola, submetidos a diferentes períodos de imersão a $25^{\circ} \mathrm{C}$ : 25 e $50 \mathrm{~mL}$ 
Ocorreram algumas variações na classificação dos lotes, quando se compararam os dados de condutividade elétrica com a germinação e os testes de vigor utilizados para a caracterização do perfil dos lotes. Porém, todos os testes destacaram o lote 4 como sendo o de qualidade inferior, com o sistema de membranas celulares menos íntegro ou com menor capacidade de restabelecer sua integridade, durante a embebição, evidenciado pela maior lixiviação de eletrólitos.

Por meio da análise de correlação simples entre as variáveis do teste de condutividade elétrica e dos testes que caracterizaram inicialmente os lotes (Tabela 3), verifica-se que a maior correlação encontrada foi entre o teste de condutividade elétrica e o teste de emergência em areia, com de correlação significativa de -0,65 em 25 mL.

A classificação dos lotes em classes de vigor foi mais evidente, nas combinações do teste de condutividade elétrica, nos períodos de 8, 16 e 24 horas em 25 mL (Tabela 2). Nestas combinações, constataram-se leituras de condutividade elétrica mais próximas das do teste de emergên- cia. Portanto, o teste de condutividade elétrica poderia ser executado com a utilização de 50 sementes imersas em $25 \mathrm{~mL}$ por 8, ou 16 ou 24 horas.

O período de 24 horas de imersão das sementes é, em geral, o recomendado para o teste de condutividade elétrica (Hampton \& Tekrony, 1995; Vieira \& Krzyzanowski, 1999); porém, a possibilidade da redução deste período é vantajosa para a indústria de sementes, pois nos seus programas de controle de qualidade buscam-se informações sobre o vigor dos lotes em período de tempo relativamente curto, como 8 ou 16 horas.

Em relação a estes períodos de imersão, verificou-se, também, que, no período de 8 horas de imersão, já foi possível separar os lotes 1 e 2 dos lotes 3 e 4, mostrando que esses lotes, mesmo num curto período de imersão, apresentaram maior potencial fisiológico, o que ratifica a vantagem de se obterem resultados que avaliem a qualidade fisiológica das sementes rapidamente, pois encurta-se, assim, o período de tomada de decisão da indústria de sementes (Marcos Filho et al.,1990; Dias \& Marcos Filho, 1996).

Tabela 3. Coeficientes de correlação simples entre os valores do teste de condutividade elétrica, nos volumes de água de 25 e $50 \mathrm{~mL}$, e os testes de germinação (G), primeira contagem (PC), comprimento de plântula (CP) e emergência em areia (EA)

\begin{tabular}{lcccc}
\hline Volume de água & $\mathbf{G}(\mathbf{\%})$ & $\mathbf{P C}(\mathbf{\%})$ & $\mathbf{C P}(\mathbf{c m})$ & $\mathbf{E A}(\mathbf{\%})$ \\
\hline 25 & $-0,63$ & $-0,55$ & $-0,59$ & $-0,65$ \\
& $(0,0027) *$ & $(0,0120)$ & $(0,0063)$ & $(0,0019)$ \\
\hline 50 & $-0,53$ & $-0,45$ & $-0,51$ & $-0,56$ \\
& $(0,0169)$ & $(0,0443)$ & $(0,0212)$ & $(0,0099)$ \\
\hline
\end{tabular}

* P-valor.

\section{CONCLUSÕES}

O teste de condutividade elétrica é eficiente para avaliação do potencial fisiológico de sementes de canola, sob a condição de 50 sementes imersas em 25 mL de água deionizada, por oito horas.

\section{REFERÊNCIAS}

Ávila RM, Braccini AL, Scapim CA, Martorelli DT \& Albrecht LP (2005) Teste de laboratório em sementes de canola e a correlação com a emergência das plântulas em campo. Revista Brasileira de Sementes, 27:62-70.

Baier AC \& Roman ES (1992) Informações sobre a cultura da “canola” para o Sul do Brasil. In: Seminário Estadual de Pesquisa de Canola, Cascavel. Anais, EMBRAPA/CNPT. p.1-10.

Bewley JD \& Black M (1994) Seeds: Physiology of development and germination. $2^{\text {a }}$ ed. New York, Plenum Press. 443p.

Brasil (2009) Regras para análise de sementes. Brasília, Ministério da Agricultura, Pecuária e Abastecimento/ACS. 399p.

Carvalho NM \& Nakagawa J (2000) Sementes: Ciência, tecnologia e produção. $4^{\text {a }}$ ed. Jaboticabal, FUNEP. 424p.

Delouche JC \& Baskin CC (1973) Accelerated aging techniques for predicting the relative storability of seed lots. Seed Science and Technology, 1:427-452.
Dias DCFS \& Marcos Filho J (1996) Testes de condutividade elétrica para avaliação do vigor de sementes de soja (Glycine max L.). Scientia Agricola, 53:31-42.

Dutra A \& Vieira RD (2006) Teste de condutividade elétrica para a avaliação do vigor de sementes de abobrinha. Revista Brasileira de Sementes, 28:117-122.

Hampton JG \& Tekrony BM (1995) Handbook of vigor methods. In: International Seed Testing Association. $3^{\text {a }}$ ed. Zürich, ISTA. p.22-34.

Ista - International Seed Testing Association (2006) International rules for testing seeds. Seed Science and Technology, 32:403.

Lima Neto AF, Santos LSS, Moura EM \& Moura CVR (2006) Biodiesel de mamona obtido por via etílica. Disponível em: <http://www.biodiesel.gov.br/docs/congressso 2006/producao/ Via_Etilica03.pdf> Acessado em: 25 de julho de 2010.

Marcos Filho J, Silva WR, Novembre ADLC \& Chama HCPC (1990) Estudo comparativo de métodos para avaliação da qualidade fisiológica de sementes de soja, com ênfase ao teste de condutividade elétrica. Pesquisa Agropecuária Brasileira, 25:1805-1815.

Marcos Filho J (1999) Testes de vigor: Importância e utilização. In: Krzyzanowski FC, Vieira RD \& França Neto JB (Eds.) Vigor de sementes: Conceitos e testes. Londrina, ABRATES. p.1-21.

Marcos Filho J (2005) Fisiologia de sementes de plantas cultivadas. $1^{\text {a }}$ ed. Piracicaba, Fundação de Estudos Agrários "Luiz de Queiroz”. 495p. 
Mello SC, Spínola MCM \& Minami K (1999) Métodos de avaliação da qualidade fisiológica de sementes de brócolos. Scientia Agrícola, 56:1151-1155.

Menezes NL, Garcia DC, Bahry CA \& Mat-tioni NM (2007) Teste de condutividade elétrica em aveia preta. Revista Brasileira de Sementes, 29:138-1427.

Nakagawa J (1994) Testes de vigor baseados na avaliação das plântulas. In: Vieira RD \& Carvalho NM (Eds.) Testes de vigor em sementes. Jaboticabal, FUNEP. p.49-85.

Nakagawa J (1999) Testes de vigor baseados no desempenho das plântulas. In: Krzyzanowski FC, Vieira RD \& França Neto JB (Eds.) Vigor de sementes: conceitos e testes. Londrina, ABRATES. p.2.1-2.24.

NTIA/EMBRAPA (1989) Núcleo tecnológico para informática. SOC - Software científico. Campinas.

Tomm GO (2007) Indicativos tecnológicos para a produção de canola no Rio Grande do Sul. Disponível em: <http:// www.cnpt.embrapa.br/culturas/canola/p_sp03_2007.pdf $>$ Acessado em: 15 de julho de 2010.
Vanzolini S \& Nakagawa J (1998) Teste de condutividade elétrica em genótipos de sementes de amendoim. Revista Brasileira de Sementes, 20:178-183.

Vidigal DS, Lima JS, Bhering MC \& Dias DCFS (2008) Teste de condutividade elétrica para semente de pimenta. Revista Brasileira de Sementes, 30:168-174.

Vieira RD, Panobiano M, Lemos LB \& Fornasieiro Filho D (1996) Efeito de genótipos de feijão e de soja sobre os resultados da condutividade elétrica de sementes. Revista Brasileira de Sementes, 18:220-224.

Vieira RD \& Krzyzanowski FC (1999) Teste de condutividade elétrica. In: Krzyzanowski FC, Vieira RD \& França Neto JB (Eds.) Vigor de sementes: conceitos e testes. Londrina, ABRATES. p.1-26. 